\title{
Breakthrough in Heart Failure therapy: LCZ696 combining ACE-Neprilysin inhibition
}

\author{
Sandeep Lahiry ${ }^{1}$, Rajasree Sinha ${ }^{2}$, Shouvik Choudhury ${ }^{3}$, Ayan Mukherjee ${ }^{4}$ \\ ${ }_{1,3,4}$ Post Graduate Trainee, Department of Pharmacology, Institute of Post Graduate Medical Education \\ and Research, ${ }^{2}$ Post Graduate Trainee, Department of Pediatrics, Medical College and Hospital, Kolkata, \\ West Bengal, India
}

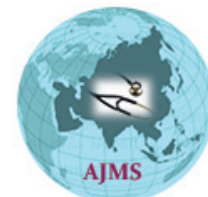

A B S T R A C T

Background: Current Heart failure (HF) pharmacotherapy has been unsatisfactory in halting disease progression completely. Aims and Objective: To evaluate the role of LCZ696, a recent FDA-approved ACE -Neprilysin inhibitor (ARNi) in the management of HF from available trial data. Materials and Methods: Trial data on 'LCZ696' was assessed using PubMed search. Methodological filters were applied to limit retrieval to 'Randomized Controlled Trial' (RCT). Bibliographic databases with 'Human' data were selected. Trial data comparing 'LCZ696' to other drugs or placebo were accessed in full-text. CONSORT guidelines were used for quality assessment. Incomplete methodology, results in abstract form, duplicate publications were excluded. Data extraction forms were piloted and used to obtain uniform quality of data. Results: Multi-centric trial data $(n=2)$ revealed noticeable benefits with 'LCZ696' in patients with HF with reduced ejection fraction (HFrEF), reducing cardiovascular death or hospitalization for HF by $20 \%$; cardiovascular deaths by $20 \%$; hospitalization for HF by $21 \%$; all cause mortality reduction by $20 \%$ as compared to ACE inhibitors (ACEi) (PARADIGM-HF; $\mathrm{n}=8442$ ). Angioedema was notably absent. Decrease in high sensitivity Troponin- $\mathrm{T}$, improvement in N-terminal-pro-BNP and left atrial dimensions suggested reduction of myocardial injury in HF with preserved ejection fraction (HFpEF) (PARAMOUNT trial; $\mathrm{n}=301$ ). Conclusion: There is convincing evidence of the role of novel ARNi (Angiotensin receptor - Neprilysin Inhibitors) in HF pharmacotherapy. Its role in other cardiovascular conditions merits assessment.

Access this article online

Website:

http://nepjol.info/index.php/AJMS DOI: 10.3126/ajms.v8i1.15861 E-ISSN: 2091-0576 P-ISSN: 2467-9100

Key words: LCZ696, Neprilysin, Heart failure

\section{INTRODUCTION}

Currently, renin-angiotensin-aldosterone system (RAAS) blockade remains the mainstay of HF pharmacotherapy. However, combination of RAAS blockade and 'Neprilysin' inhibition, an enzyme that degrades natriuretic peptides (NPs), has recently emerged as a potentially superior treatment strategy. On 7th July 2015, Valsartan-Sacubitril (LCZ696) received FDA's priority approval for use in $\mathrm{HF}^{1}$ In retrospect, the approval was based on data from the Prospective Comparison of ARNi [angiotensin receptor-neprilysin inhibitor] with ACEi to Determine Impact on Global Mortality and Morbidity in Heart Failure (PARADIGM-HF; NCT01035255
Funded by Novartis) trial, when it was presented at the European Society of Cardiology (ESC) 2014 Congress at Barcelona, Spain. ${ }^{2}$

\section{MATERIALS AND METHODS}

\section{Literature search}

Online literature was assessed using PubMed search. Search strategy consisted of controlled vocabulary and keywords. Main search concepts were 'LCZ696', 'Heart failure' and 'Neprilysin'. Methodological filters were applied to limit retrieval to 'Randomized Controlled Trial' (RCT). Retrieval was limited to 'English' and 'Human'. 


\section{Selection criteria \& methodology}

Reviewers independently screened citations of preliminary selected RCTs $(\mathrm{n}=6)$ and current clinical practice guidelines on HF. In cases of insufficient information, full-text article with abstract was ordered. Only full-text publications were inlcuded. Additional information was accessed using Google Scholar search engine.

\section{Exclusion criteria}

Studies with incomplete methodology; presented preliminary results in abstract form; duplicate publications, editorials were excluded.

\section{Data extraction strategy}

Data extraction forms were piloted and independent extraction of clinical effectiveness data for each article was undertaken. Relevant features \& outcomes from the included studies were tabulated. Any disagreement between reviewers was discussed until consensus was reached.

\section{Critical appraisal of individual studies}

Quality of RCTs was assessed following CONSORT guidelines.

\section{Data analysis method}

Because of clinical heterogeneity across the selected studies, a formal analysis was not conducted. The findings are described using a narrative approach.

\section{RESULTS}

Electronic literature search yielded a total of 76 citations, of which 25 citations were excluded, and 51 potentially relevant articles were shortlisted. 12 articles were available for full-text review, of which 6 were RCTs. Complete data on 2 RCTs primarily concerning to HF therapy were included (PARAMOUNT and PARADIGM-HF).

The PARADIGM-HF study demonstrated the favorable effect of LCZ696 that was seen in all subgroups examined, including those based on age, sex, weight, race, NYHA class, presence or absence of reduced kidney function, prior hospitalization etc. Significant benefits were recorded in patients with NYHA class II-IV Systolic HF using 'LCZ696' over ACE inhibitors (ACEi), reducing cardiovascular death or hospitalization for HF by $20 \%$, cardiovascular deaths by $20 \%$, hospitalization for Heart Failure by $21 \%$ and all-cause mortality reduction by $20 \% .^{3}$ Patients were followed up for an average of 27 months. The study revealed LCZ696 to be superior to enalapril in a well-managed cohort of patients with HF, $93 \%$ with $\beta$-adrenergic blockers therapy and $55.6 \%$ on mineralocorticoid receptor antagonists (MRA). ${ }^{4}$
The PARAMOUNT (Prospective Comparison of ARNi with ARB on Management of Heart Failure with Preserved Ejection Fraction: NCT01920711) trial compared LCZ696 with valsartan in patients $(\mathrm{n}=301)$ having $\mathrm{HF}$ with preserved ejection fraction (HFpEF). ${ }^{5}$ There was a significant decrease in NT-proBNP (N-terminal of Brain natriuretic peptide) levels in the LCZ696 group at 12 weeks; however, the difference was insignificant at 36 weeks. Furthermore, there was no change in LV size, function, or mass; diastolic function; NYHA class; or quality-of-life scores at 12 weeks. ${ }^{5}$

\section{DISCUSSION}

Natriuretic peptides (NP) are hormones responsible for maintaining normal sodium and fluid homeostasis. Their release is triggered by increased myocardial filling pressures. ${ }^{6}$ Their beneficial role include vasodilation, increased glomerular filtration rate (GFR) and reduced renal release of renin, in addition to natriuresis and diuresis. ${ }^{7}$ NPs are broken down by an enzyme neutral endopeptidase (NEP) or 'Neprilysin'. ${ }^{8}$

Neprilysin, a metalloprotease (zinc-dependent), cleaves hydrophobic peptide residues at the amino side and inactivates numerous peptides. ${ }^{9}$ It also degrades the amyloid-beta peptide whose abnormal misfolding and aggregation in neural tissue implicated to be causative of Alzheimer's disease. ${ }^{10}$ Degradation of vasoactive substances like peptides, glucagon, enkephalins, substance P, neurotensin, oxytocin, and bradykinin and adrenomedullin by Neprilysin, reduces deleterious neurohormonal activation, subsequent vasoconstriction, sodium retention and maladaptive remodeling. ${ }^{11}$

Increased salutary NP actions also have a beneficial role in HF. Neprilysin is known to hydrolyze NPs, therefore its inhibition would mitigate risk of cardiovascular disease. When the left ventricle and myocardium is stressed as in $\mathrm{HF}$, there is an increase in diastolic pressure wall stress; the myocytes pro-BNP which is cleaved in two subunits in the plasma: the inactive $\mathrm{N}$-terminal fragment and the active C-terminal fragment, BNP. ${ }^{12}$ In the PARADIGM-HF trial, in patients with HFrEF, LCZ696 caused reduction in NT-proBNP to a greater extent than did valsartan at 12 weeks and was better tolerated. ${ }^{13}$

Inhibiting Neprilysin had been a therapeutic target for several compounds that had previously been tested in cardiovascular disease, including ecadotril, candoxatril, omapatrilat and other NEPi-ACEi (vasopeptidase-inhibitors). ${ }^{14}$ Although these drugs were initially tested in their role in hypertension and/or HF, lack of efficacy and side effects (Angioedema) led to discontinuation of their development. ${ }^{15}$ 
LCZ696 (Valsartan-Sacubitril) is a novel ARNi developed and approved for use in HF. It has six molecules of the Neprilysin inhibitor Sacubitril (AHU-377), six molecules of anionic form of Valsartan bound together in a single crystalline complex. ${ }^{16}$ Valsartan moiety blocks the angiotensin II receptor type 1 (AT1) and thereby causing vasodilation and increased excretion of sodium and water via the kidneys (by reducing aldosterone production). The latter mechanism also causes a reduction in blood volume. Sacubitril, a prodrug, is activated to LBQ657 by de-ethylation via esterases. ${ }^{17} \mathrm{LBQ} 657$ causes inhibition of enzyme Neprilysin, which is responsible for the degradation of ANP \& BNP, two blood pressure lowering peptides that work mainly by reducing blood volume. Because this molecule is made in a fixed ratio (1:1), the $97 \mathrm{mg}$ of Sacubitril provides about $90 \%$ Neprilysin inhibition. ${ }^{18}$ It has been proposed as a twice daily regimen, costing $\$ 12.50$ /day (Entresto ${ }^{\circledR}-$ Novartis) in U.S.A, can be taken regardless of food intake. ${ }^{19}$ Experts are enthusiastic about the prospects of the ARNi, believed to be representing the future cornerstone of chronic HF therapy. The new Canadian Heart Failure guidelines have already been updated to include the drug in its recommendations. ${ }^{20}$

Though recent guidelines have started incorporating ARNi in HFrEF pharmacotherapy, complete status of LCZ696 in management of newly diagnosed HFpEF patients remains yet unclear. Though expected to show better compliance and favourable adverse effect profile, complete data on safety and tolerability profile will be assessed further following its use in a broad range of patients with HF with different symptomatic spectrum. Long term trials may still be needed to consolidate its decisive role.

\section{ACKNOWLEDGEMENT}

We are extremely thankful to the faculty of the Department of Pharmacology, Institute of Post Graduate Medical Education and Research, Kolkata for their kind permission to carry out this study.

\section{Key message}

LCZ696 opens up a new class of novel ARNi, which seeks to exploit clinical efficacy of combined RAAS-blockade and NEPi-mediated natriuretic peptide augmentation with improved clinical safety profile \& seems to challenge existing evidence-based medical therapy in HF.

\section{REFERENCES}

1. Gibney M. "Novartis' new heart failure medicine LCZ696 approved by FDA to reduce risk of cardiovascular death and heart failure hospitalization; now called Entresto ${ }^{\mathrm{TM}}$ (sacubitril/ valsartan)". FierceBiotech. 2015.

2. Lim GB. Heart failure: LCZ696--a PARADIGM shift in treatment for heart failure. Nat Rev Cardiol 2014;11(11):618.

3. McMurray JJ, Packer M, Desai AS, Gong J, Lefkowitz M, Rizkala AR, et al. Baseline characteristics and treatment of patients in prospective comparison of ARNI with ACEI to determine impact on global mortality and morbidity in heart failure trial (PARADIGM-HF). Eur J Heart Fail 2014; 16(7):817-825.

4. McMurray JJ, Packer M, Desai AS, Gong J, Lefkowitz MP, Rizkala AR, et al. Dual angiotensin receptor and neprilysin inhibition as an alternative to angiotensin-converting enzyme inhibition in patients with chronic systolic heart failure: rationale for and design of the Prospective comparison of ARNI with ACEI to Determine Impact on Global Mortality and morbidity in Heart Failure trial (PARADIGM-HF). Eur J Heart Fail 2013; 15(9):1062-1073.

5. Jhund PS, Claggett B, Packer M, Zile MR, Voors AA, Pieske B, et al. Independence of the blood pressure lowering effect and efficacy of the angiotensin receptor neprilysin inhibitor, LCZ696, in patients with heart failure with preserved ejection fraction: an analysis of the PARAMOUNT trial. Eur J Heart Fail 2014; 16(6):671-677.

6. Burnett JC, Kao PC, Hu DC, Heser DW, Heublein D, Granger JP, et al. Atrial natriuretic peptide elevation in congestive heart failure in the human. Science 1986; 231(4742):1145-1147.

7. Maack $T$, Suzuki $M$, Almeida FA, Nussenzveig D, Scarborough RM, McEnroe GA, et al. Physiological role of silent receptors of atrial natriuretic factor. Science 1987; 238(4827):675-678.

8. de Lemos JA, McGuire DK and Drazner MH. B-type natriuretic peptide in cardiovascular disease. Lancet 2003; 362(9380):316-322.

9. Turner AJ, Isaac RE and Coates D. The neprilysin (NEP) family of zinc metalloendopeptidases: genomics and function. Bioessays 2001; 23(3):261-269.

10. Yasojima K, Akiyama H, McGeer EG and McGeer PL. Reduced neprilysin in high plaque areas of Alzheimer brain: a possible relationship to deficient degradation of beta-amyloid peptide. Neurosci Lett 2001; 297(2):97-100.

11. Oefner C, D'Arcy, Hennig, Winkler F and Dale GE. Structure of human neutral endopeptidase (Neprilysin) complexed with phosphoramidon. J of Mol Bio 2000; 296(2):341-349.

12. Cowie MR and Mendez GF. BNP and congestive heart failure. Prog Cardiovasc Dis 2002; 44(4):293-321.

13. Solomon SD, Zile M, Pieske B, Voors A, Shah A, Kraigher-Krainer E, et al. The angiotensin receptor neprilysin inhibitor LCZ696 in heart failure with preserved ejection fraction: a phase 2 double-blind randomised controlled trial. Lancet 2012; 380 (9851):1387-1395.

14. Basuray I. Neutral peptidase inhibitors: New drugs for heart failure. Indian J of Pharmacol 2003; 35(3): 139-145.

15. Bralet J and Schwartz JC. Vasopeptidase inhibitors: an emerging class of cardiovascular drugs. Trends Pharmacol Sci 2001; 22(3):106-109.

16. Gu J, Noe A, Chandra P, Al-Fayoumi S, Ligueros-Saylan M, Sarangapani R, et al. Pharmacokinetics and pharmacodynamics of LCZ696, a novel dual-acting angiotensin receptor-neprilysin inhibitor (ARNi). J Clin Pharmacol 2010; 50(4):401-414.

17. Loretta Fala. Entresto (Sacubitril/Valsartan): First-in-Class Angiotensin Receptor Neprilysin Inhibitor FDA Approved for Patients with Heart Failure. Am Health Drug Benefits 2015; 8(6): 330-334.

18. Flarakos J, Du $\mathrm{Y}$, Bedman $\mathrm{T}$, Al-Share $\mathrm{Q}$, Jordaan $\mathrm{P}$, Chandra $P$, et al. Disposition and metabolism of [(14) 
C] Sacubitril/Valsartan (formerly LCZ696) an angiotensin receptor neprilysin inhibitor, in healthy subjects. Xenobiotica 2016; 46(11):986-1000.

19. Senior M. Heart drug pushes outcome-based pricing plans. Nat Rev Drug Discov. 2015; 14(10):665-667.
20. Moe GW, Ezekowitz JA, O'Meara E, Lepage S, Howlett JG, Sussex B, et al. The 2014 Canadian Cardiovascular Society Heart Failure Management Guidelines Focus Update: anemia, biomarkers, and recent therapeutic trial implications. Can J Cardiol 2015; 31(1):3-16.

Authors Contribution:

SL - Reviewed the literature and manuscript preparation; SC - Collected data, review of literature and helped in preparing first draft of manuscript;

AM - Literature search and critical revision of the manuscript; RS - Concept of study and review of study. 